\title{
Simulation of Dimple Characteristics on the Trajectories of a Dimpled Sphere (Golf Ball) in Motion
}

Wainaina $\mathbf{M}^{1 *}$, Kimathi $\mathbf{M}^{2}$ and Malonza $D^{3}$

${ }^{1}$ Catholic University of Eastern Africa, Nairobi, Kenya

${ }^{2}$ Technical University of Kenya, Nairobi, Kenya

${ }^{3}$ Kenyatta University, Nairobi, Kenya

\begin{abstract}
A mathematical model describing the flight motion of a golf ball is developed, and the effects of dimple characteristics are studied. Using the Newton's second law of motion, the equations governing the motion of the golf ball are developed in three dimensions. In this development the size, depth and number of dimples are taken into account. By varying the dimple size, depth and number, the effects of these characteristics are simulated via a MatLab code in which the Dormand-Prince Runge Kutta method is implemented to solve the model equations. The results of the numerical simulations that show how the golf ball trajectory is influenced by the dimple characteristics such as dimple depth, size and number within the accepted range of the Reynolds number are displayed and discussed.
\end{abstract}

Keywords: Dimple; Trajectories; Boundary layer; Reynolds's number

\section{Introduction}

Mathematical research in golf is of great interest because of the uniqueness of the golf ball. Many researchers have not ventured in this field which carries a lot of mathematical concepts [1]. Flight Trajectory which is a core term in golf refers to the path a ball takes after impact, while it is in the air. Besides the straight lines trajectories there are other types of trajectories namely: a pull, push, hook and slice as shown in Figure 1. A pull is when the ball starts its path to the left of its intended destination without spinning sideways while a push results when the ball starts its path to the right of its intended destination, also without spinning sideways [2-4]. A hook is a ball flight in which the ball curves from right to left due to a small amount of side-spin being imparted to it at impact. Similarly, a slice is a ball flight in which the ball curves from left to right, due to side-spin imparted to the ball at impact in the opposite direction from the one used to obtain the hook result [5-11]. Each of these terms is defined for a player using "right-handed" clubs, and changes meaning if the player uses "lefthanded" clubs.

Dimples are small indentations on the exterior of the golf ball. They are typically round in shape and vary in diameter from $2-5 \mathrm{~mm}$ and are about $0.2 \mathrm{~mm}$ deep. Modern golf balls have about 300-450 dimples of varying size arranged in a regular pattern on the surface of every ball. Dimples have been one of the most influential developments in golf ball design because they alter the dynamics of the ball's flight in a way that gives golfers a significant amount of control over the height and shape of their shots. Some golf balls have patterns of round dimples but most manufacturers produce balls with dimple patterns in a variety of hexagonal shapes; the most common dimple patterns are the icosahedral, the dodecahedral, and the octahedral (Figure 2). The icosahedral pattern is based on a polyhedral with 20 identical triangular faces, much like a 20 -sided die. Similarly, a dodecahedral is based on a polyhedral with 12 identical faces in the shape of pentagons. The octahedral is based on an 8-sided polyhedral with triangular faces. Some balls with icosahedral pattern have up to 500 dimples. Moreover, in balls such as the Nike PD2 shown in Figure 2, all the dimples are not of the same diameter; this is done with the sole aim of improving the ball's performance during flight.
The depth and size of the dimples also affect the golf ball performance. Shallow dimples generate more spin on the golf ball than deep dimples. . On the other hand, deep dimples make a golf ball to spin less as compared to shallow dimples. Shallow dimples generally give the ball a lower trajectory and good control in the wind; whereas large dimples give the ball a higher trajectory and longer flight time [1-4]. Naruo and Mizota [5] worked on the influence of golf ball dimples on aerodynamic behaviour of the airflow around the ball using a wind tunnel and rotating device. By using golf balls whose dimples

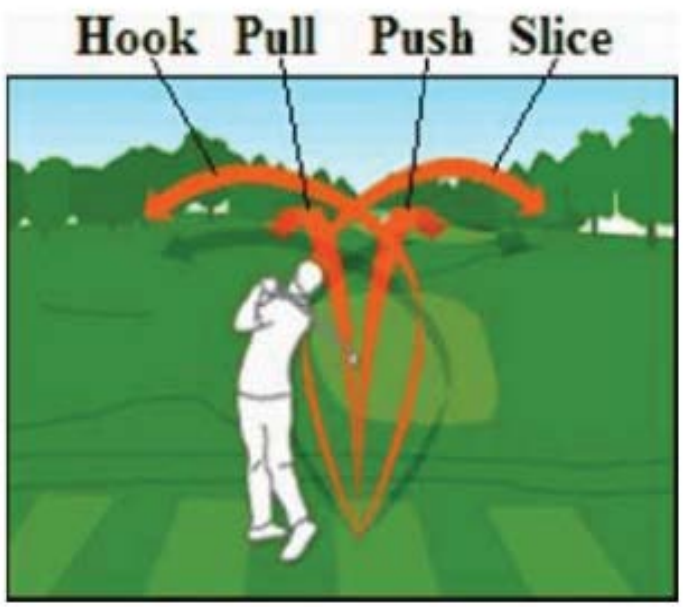

Figure 1: The possible flight trajectories which depend on how the golf ball is struck.

*Corresponding author: Wainaina M, Catholic University of Eastern Africa, Nairobi, Kenya, Tel: +254 724 253733; E-mail: nyawiragitoi@gmail.com

Received September 11, 2017; Accepted February 27, 2018; Published March 06, 2018

Citation: Wainaina M, Kimathi M, Malonza D (2018) Simulation of Dimple Characteristics on the Trajectories of a Dimpled Sphere (Golf Ball) in Motion. J Appl Computat Math 7: 386. doi: 10.4172/2168-9679.1000386

Copyright: @ 2018 Wainaina M, et al. This is an open-access article distributed under the terms of the Creative Commons Attribution License, which permits unrestricted use, distribution, and reproduction in any medium, provided the original author and source are credited. 


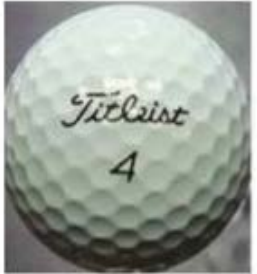

(a) Titleist Pro V1

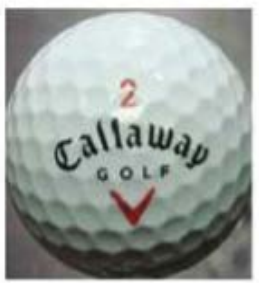

(e) Callaway Big Bertha

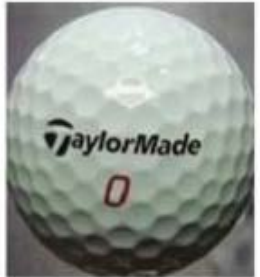

(b) TaylorMade TP/Red LDP

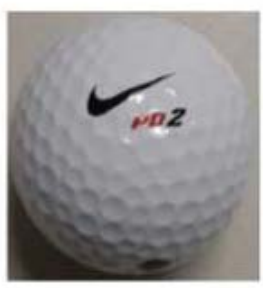

(f) Nike PD 2

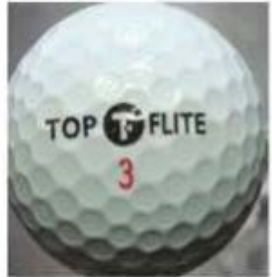

(c) Top Flite D2 Distance

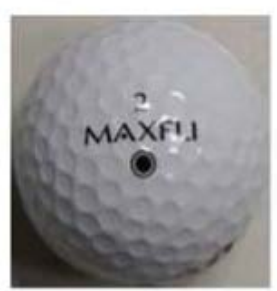

(g) MaxFi

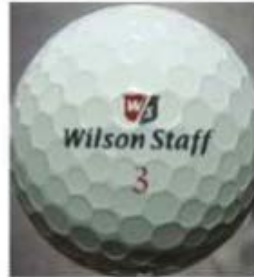

(d) Wilson Staff DX2

Distance

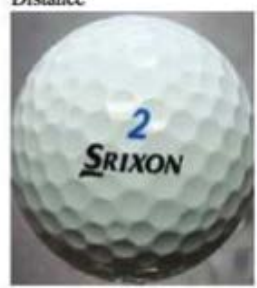

(b) Srixon AD333

Figure 2: Range of golf balls with different dimple patterns:(Firoz et al. (2011)).

have different depths, they found that shallower dimples resulted to the larger lift coefficient. However, when the depth of the dimples was much shallower, the lift coefficient was extremely small for slow winds of velocities below $30 \mathrm{~m} / \mathrm{s}$. The consequence of a small lift (coefficient) is that distance covered by the ball will be short. Additionally, dimple patterns that had a high lift coefficient at all velocities were researched by these authors. As a result, it was found that a golf ball with a dimple pattern that has extremely small dimples between large shallow dimples has a high lift coefficient at all velocities, including those below $30 \mathrm{~m} / \mathrm{s}$. Visualization of flow around a golf ball was conducted by introducing smoke in the experiment set-up and using a high-speed video camera to take pictures that were analysed by PIV (Particle Image Velocimetry). From the pictures a difference in the streamline distribution was noted for different dimple depths on the golf balls.

\section{Layer separation and the drag force}

A smooth (golf) ball will generate a large wake (the region of recirculating flow immediately behind a moving or a stationary blunt body) behind the ball, as there is much lower pressure behind the ball than in the front. The air will rush into the area of low pressure, exerting a pressure drag force on the smooth ball. This drag force hinders the forward motion of the ball since it acts in the direction opposite to that of ball motion. Therefore, the purpose of having a large number of dimples on a golf ball is based on the Euler's principle which states that separation of the boundary layer (the layer of air molecules next to the surface of the golf ball) is likely to occur in regions where the pressure increases in the direction of the flow. So introduction of dimples on the golf ball ensures that the boundary layer does not separate until at the back part of the ball, resulting into a thin wake as shown in Figure 3.

\section{Significance of the Reynolds number in the ball aerodynamics}

In fluid flows, the Reynolds number (Re) is a dimensionless number that gives a measure of the ratio of inertial forces to viscous forces for some given flow conditions. The Reynolds number is an important parameter that describes whether flow conditions lead to laminar or turbulent flow. Laminar flow occurs when a fluid flows in parallel layers, with no disruption in the layers. Turbulent flow is a flow regime characterized by chaotic property changes. It is significant in the design of a model of any system in which the effect of viscosity is important in controlling the velocities or the flow pattern of a fluid and given by

$$
\operatorname{Re}=\frac{\rho V L}{\mu}
$$

Where

$\rho=$ the density of the fluid;

$V=$ the velocity of the fluid;

$\mu=$ the viscosity of the fluid;

$\mathrm{L}=$ the length or diameter of the fluid.

The drag coefficient encountered in the drag force during the development of our governing equations of the flight of the ball, depends on the Reynolds number. This dependence is created because as stated above the Reynolds number determined the transition from laminar to turbulent regimes. This transition occurs at $\operatorname{Re} \approx 3 \times 105$, where a sharp drop in drag coefficient is experienced. Note that flows characterized by low Reynolds numbers are slow and orderly (laminar), whereas those characterized by high Reynolds number are fast and chaotic (turbulent). Since the golf ball we consider in our model development is dimpled and because the boundary layer changes from laminar to turbulent much sooner, we use a high value of the Reynolds number in the simulations.

In this study we address the modelling of the flight trajectory in three dimensions and taking in to account the empirical findings of the effects of dimple characteristics that has been achieved on aerodynamic behaviour. The main aim is to produce a mathematical model and perform numerical simulations of the trajectory of a golf ball in motion while taking into consideration the dimples and spin effects.

\section{Model Formulation}

The appropriate way of describing the trajectory of a golf ball is to take it as an object in motion and as such that, it then obeys the Newton second law of motion. This law states that the acceleration of an object depends on two things namely: the net forces acting upon the object and mass of the object. The forces that influence the flight motion of a golf ball are shown in Figure 4 and described briefly as below: 


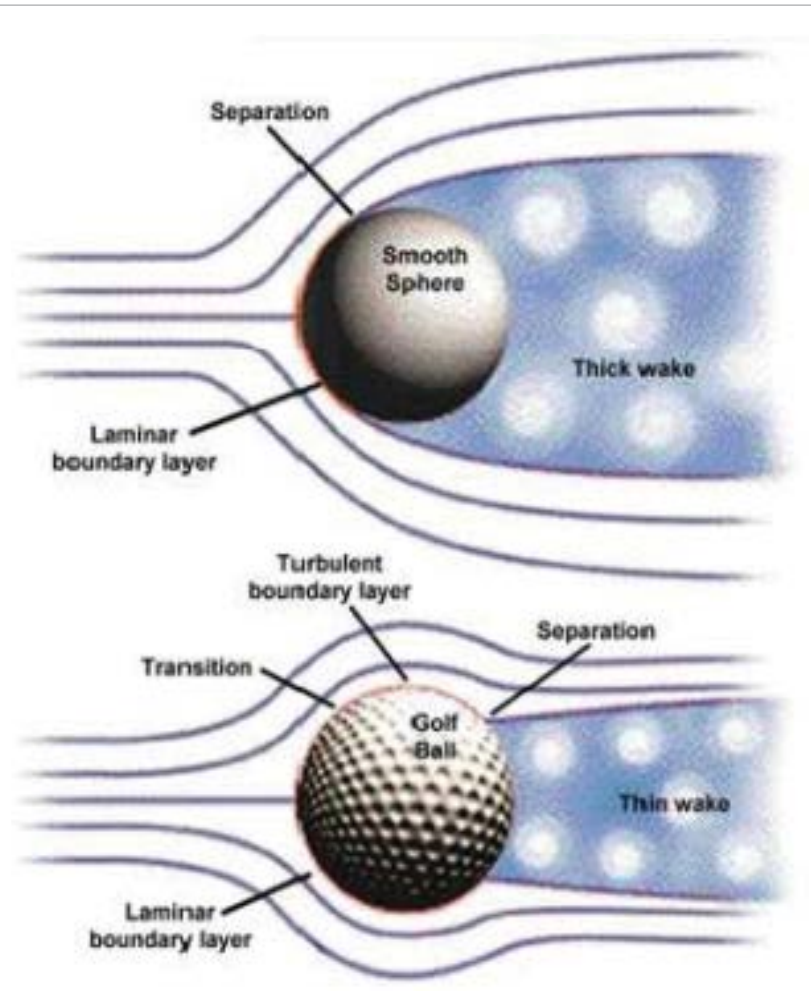

Figure 3: Flow separation in a smooth sphere and a dimpled sphere respectively in motion:(http://www.aerospaceweb.org).

\section{Drag force $\vec{F}_{\text {Drag }}$}

As the ball penetrates through the air, it pushes air molecules out of the way, whereby the air molecules apply a force equal in magnitude but in a direction opposite to the direction of motion of the ball as shown in Figure 4 , whereby the ball is moving in the direction indicated by $\vec{V}$.

Suppose that the variables involved under some conditions are speed, Fluid Density, viscosity of the fluid, size of the body, expressed in terms of the frontal area and drag force. These five variables can be reduced to two dimensionless parameters: Drag coefficient $C_{d}$ and Reynolds number Re.

When the drag force is expressed as part of a function of the other variables in the problem, we can have the expression:

$$
\mathrm{f}_{\mathrm{a}}\left(\mathrm{f}_{\mathrm{d}}, \mathrm{V}, \mathrm{A}, \rho, \mathrm{v}\right)=0
$$

Here $\mathrm{f}_{\mathrm{a}}=\mathrm{a}$ function that takes five arguments.

Now taking the right hand side to be zero in any system of units it is possible to express the relationship described in terms of only dimensionless groups using the Buckingham $\pi$ theorem in dimensional analysis. In this case the two groups are the Reynolds number

$$
\mathrm{Re}=\frac{V \sqrt{A}}{v}
$$

And the drag coefficient, given by

$$
C_{d}=\frac{F_{d}}{\frac{1}{2} \rho A V^{2}}
$$

Thus the function of the five variables in eqn. (2) may be replaced by another function of only two variables using eqns. (3) and eqn. (4):

$$
f_{d}\left(\frac{F_{d}}{\frac{1}{2} \rho A V^{2}} \frac{V \sqrt{A}}{v}\right)=0
$$

Where $\mathrm{f}_{\mathrm{b}}=$ some function of two arguments.

The original equation is then reduced to an equation involving only these two groups. Because the only unknown in the above equation is the drag force $\mathrm{F}_{\mathrm{d}}$, it is possible to express it as

$$
\begin{aligned}
& \frac{F_{d}}{\frac{1}{2} \rho A V^{2}}=f_{c}\left(\frac{V \sqrt{A}}{v}\right) \\
& \text { Or } \\
& f_{d}=\frac{1}{2} \rho A V^{2} f_{c}(\mathrm{Re})
\end{aligned}
$$

And

$\mathrm{Cd}=\mathrm{f}_{\mathrm{c}}\left(\mathrm{R}_{\mathrm{e}}\right)$

by replacing $f_{c}(R e)$ with which reduces to $C d$ in eqn. (8) and noting that the drag force acts in the opposite direction of flight motion ( $\vec{V}$ ),we get the drag force equation

$$
\vec{F}_{\text {Drag }}=\frac{1}{2} \rho A C_{d}|V|^{2}(-\overrightarrow{\mathrm{V}})
$$

Where $\rho=$ the density of air at the altitude and temperature of the location where the golf ball is being played;

$A=$ the cross-sectional area of the ball;

$\vec{V}=$ the unit vector of the golf ball's instantaneous velocity, implying that the drag is constantly changing;

$\mathrm{C}_{\mathrm{d}}$-dimensionless drag coefficient.

Later, in this study we will let this coefficient depend on the dimple characteristics of the golf ball. This is because the dimples on the golf ball play a significant role in reducing the overall drag experienced by the ball during flight.

\section{Lift force}

This force acts perpendicular to the direction of motion (Figure 5). This lift force is also referred to as the Magnus force.

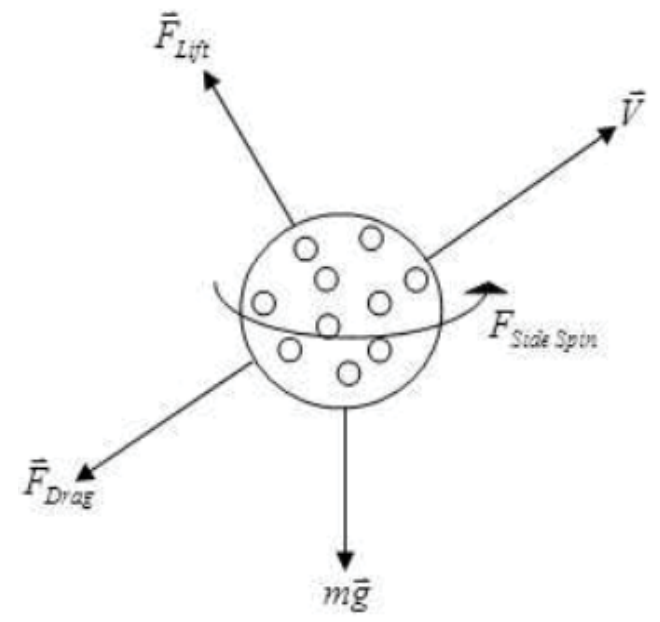

Figure 4: Forces acting on a golf ball. 
This lift force is formulated as below [6]:

$$
\vec{F}_{L i f t}=\frac{1}{2} \rho A C_{l}|V|^{2}(\overrightarrow{\mathrm{L}})
$$

Where $\rho, A, \vec{L}$ are as defined in the drag force and $C_{1}$ is a dimensionless lift coefficient which we will let to depend on the dimple characteristics of the golf ball so as to study how these characteristics influence the flight trajectory of a golf ball and $\overrightarrow{\mathrm{L}}$ is a unit vector perpendicular to ball velocity vector $\vec{F}$ and its purpose is to enable us describe the lift force vector $\vec{F}$ Lift appropriately.

The total force acting on the golf ball during flight comprises the above stated forces, that is:

$$
\vec{V}=V_{1} \hat{i}+V_{2} \hat{j}+V_{3} \hat{k}
$$

To elaborate more on the forces, we represent the forces and the unit vectors in play in a three dimensional coordinate system as illustrated in Figure 6:

Suppose that the golf ball flight trajectory is a space curve such that at some time instant, the position of the golf ball in flight is given by $(\mathrm{X}, \mathrm{Y}, \mathrm{Z})$. Suppose that at this position the ball's velocity is given by the unit vector $\vec{V}=V_{1} \hat{i}+V_{2} \hat{j}+V_{3} \hat{k}$ and its projection on the -plane is given by

$\vec{V}_{p}$. Also let $\vec{L}=L_{1} \hat{i}+L_{2} \hat{j}+L_{3} \hat{k}$ define a unit vector that is perpendicular $\vec{V} \cdot \theta$ is the angle the velocity vector makes with the $\mathrm{z}$-axis, while $\phi$ is the azimuthal angle the projection of the velocity vector makes with the $\mathrm{x}$-axis.

Here V1,V2,V3 are the golf ball's velocity components in $\mathrm{X}, \mathrm{Y}, \mathrm{Z}$ directions respectively. From calculus of vectors, these velocity components can be expressed in spherical coordinates to obtain the velocity vector as below:

$$
\vec{V}=\sin \theta \cos \varphi \hat{i}+\sin \theta \sin \varphi \hat{j}+\cos \theta \hat{k}
$$

The unit vector $\vec{V}$ is obtained by taking $\vec{V}$ and rotating it backwards and while maintaining. This means that $\vec{L}$ will coincide with $\vec{L}$ and so the angle between $\vec{L}$ and the Z-axis will be $\theta$. Using the trigonometric identities in calculus, we get that $\sin \left(\theta-\frac{\pi}{2}\right)=-\cos \theta$ and $\cos \left(\theta-\frac{\pi}{2}\right)=-\sin \theta$. These results are then substituted in eqn. (12)

To get the unit vector $\vec{L}$ as:

$$
\vec{L}=L 1 \hat{i}+L_{2} \hat{j}+L_{3} \hat{k}=-\cos \theta \cos \varphi \hat{i}-\cos \theta \sin \varphi \hat{j}+\sin \theta \hat{k}
$$

\section{Equations governing the motion of the golf ball}

In view of the golf ball motion representation in Figure 6, and using the total forces, acting on this ball, as in eqn. (11) we apply the Newton's second law of motion to yield:

$$
\begin{aligned}
& \mathrm{m} \frac{d \vec{V}}{d t}=-m \vec{g}+\vec{F}_{d r a g}+\vec{F}_{\text {lift }} \\
& \mathrm{m} \frac{d V_{1}}{d t}=0+\frac{1}{2} \rho A C_{d}|\vec{V}|^{2}\left(-V_{1}\right)+\frac{1}{2} \rho A C_{l}|\vec{V}|^{2}\left(-L_{1}\right) \\
& \mathrm{m} \frac{d V_{2}}{d t}=0+\frac{1}{2} \rho A C_{d}|\vec{V}|^{2}\left(-V_{2}\right)+\frac{1}{2} \rho A C_{l}|\vec{V}|^{2}\left(-L_{2}\right) \\
& \mathrm{m} \frac{d V_{3}}{d t}=m \vec{g}+\frac{1}{2} \rho A C_{d}|\vec{V}|^{2}\left(-V_{3}\right)+\frac{1}{2} \rho A C_{l}|\vec{V}|^{2}\left(-L_{3}\right)
\end{aligned}
$$

In eqn. (14) is the vector form representation of the equations that describe the motion of a golf ball of mass $\mathrm{m}$, in flight in 3 dimensions. Since the golf ball flight trajectory is considered to be a space curve, we can express the velocity components as:

$$
V_{1}=\frac{d X}{d t}=\dot{X}, V_{2}=\frac{d Y}{d t}=\dot{Y}, V_{3}=\frac{d Z}{d t}=\dot{Z}
$$

Such that $|\vec{V}|=\sqrt{\left(\dot{X}^{2}+\dot{Y}^{2}+\dot{Z}^{2}\right)}=1$ since $\vec{V} \mid$ is a unit vector eqn. (12). Utilizing the relations in eqn. (18) we write the eqn. (14) in component form as follows:

In X-direction;

$$
m \ddot{X}=-\frac{1}{2} \rho A C_{d}|\vec{V}|^{2}(\sin \theta \cos \varphi)+\frac{1}{2} \rho A C_{l}|\vec{V}|^{2}(-\cos \theta \sin \varphi)
$$

In Y-direction

$$
m \ddot{Y}=-\frac{1}{2} \rho A C_{d}|\vec{V}|^{2}(\sin \theta \cos \varphi)+\frac{1}{2} \rho A C_{l}|\vec{V}|^{2}(-\cos \theta \sin \varphi)
$$

In Z-direction

$$
m \ddot{Z}=-m \vec{g}-\frac{1}{2} \rho A C_{d}|\vec{V}|^{2}(\cos \theta)
$$

Note that the gravitational force acts vertically downwards and so its vector is $g=-g \hat{k}$. The above systems of eqns., (19), (20), and (21)

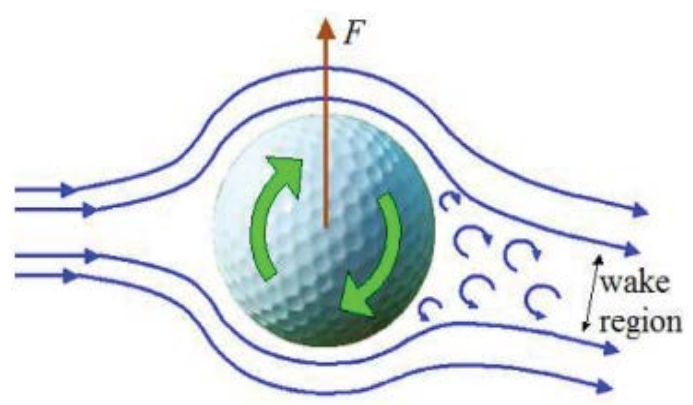

Figure 5: The lift force and the wake region (Aoki K. et al. (2009)).

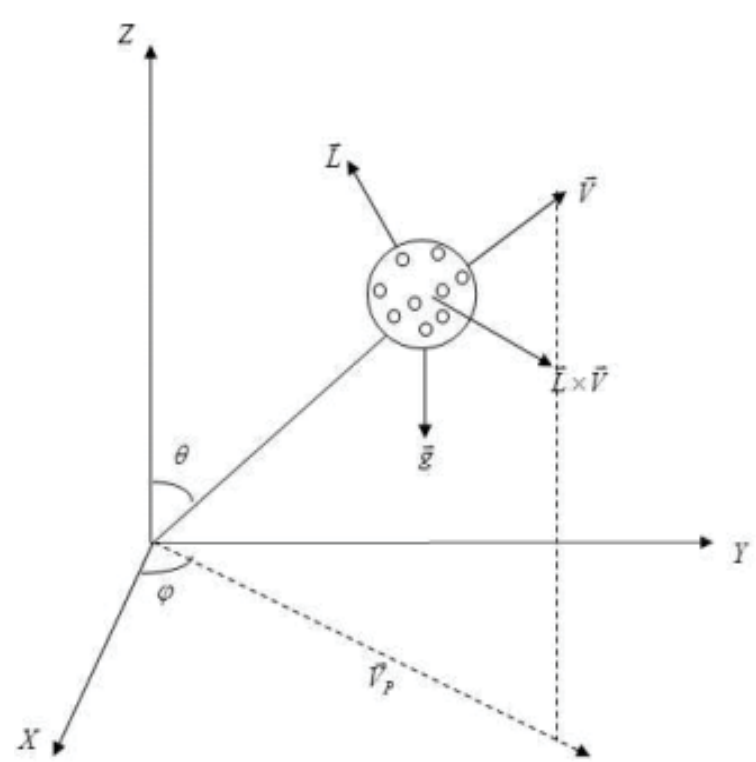

Figure 6: Three dimensional representation of the unit vectors for the forces acting on a golf ball in motion. 
can be expressed in an alternative form which we find more useful in describing the flight motion. To obtain this other form, we need to first express the sines and cosines on the right hand side of the above equations in terms of $\mathrm{X}, \mathrm{Y}$ and $\mathrm{Z}$. To this end, consider the following figures, derived from Figure 7.

Then it follows that

$$
\begin{aligned}
& \sin \theta=\frac{\left|\vec{V}_{p}\right|}{|\vec{V}|}, \cos \theta=\frac{V_{3}}{|\vec{V}|}=\frac{\dot{Z}}{|\vec{V}|} \\
& \sin \varphi=\frac{\left|V_{2}\right|}{\left|\vec{V}_{p}\right|}=\frac{\dot{Y}}{\left|\vec{V}_{p}\right|}, \cos \varphi=\frac{X}{\left|\vec{V}_{p}\right|}
\end{aligned}
$$

Substituting eqn. (22) into eqns. $(19,20$, and 21) we get the following alternative form of the equations

$$
\begin{aligned}
& \ddot{X}=-k_{d}|\vec{V}| \dot{X}-k_{1}|\vec{V}| \dot{Z} \dot{X} \\
& \ddot{Y}=-k_{d}|\vec{V}| \dot{Y}+k_{1} \frac{|\vec{V}|}{\left|\vec{V}_{P}\right|} \dot{Y} \dot{Z} \\
& \ddot{Z}=-m g-k_{d}|\vec{V}| \dot{Z}-k_{1}|\vec{V}|\left|\vec{V}_{p}\right|
\end{aligned}
$$

Where

$$
k_{i}=\frac{1}{2} \frac{\rho A C_{1}}{m} \text { f or } i \in[\mathrm{d}, 1]
$$

\section{Incorporation of the golf ball dimple characteristics into the governing equations}

The golf ball dimple size, depth and number significantly influence the flight trajectory and consequently determine how far the ball travels when played. Several experimental studies such as Naruo and Mizota [5], Baek and Kim [7], Alam et al. [8] and Aoki et al. [1-4] have shown that shallow dimples generate more spin of a golf ball than deep dimples. Additionally, these studies also revealed that smallsized dimples generally result to a lower trajectory whereas large-sized dimples result to a higher trajectory and longer flight time.

Moreover, in these experiments, it was noted that having a good number of dimples on the golf ball ensures that the laminar boundary layer around the ball becomes turbulent sooner thus resulting to a reduction in drag force that opposes the ball motion.

Due to these findings, it is essential to take into account the dimple characteristics in development of a model that describes the flight motion of a golf ball. This study aims to achieve this by decomposing the drag and lift coefficients as:

$$
\begin{aligned}
& \left.C_{d}=C_{d}(\text { size })+C_{d} \text { (number }\right)+C_{d}(\text { depth }) \\
& C_{l}=C_{l}(\text { size })+C_{1}(\text { number })+C_{1}(\text { depth })
\end{aligned}
$$

And define the contributions of the dimple size, number and depth to the drag and lift intuitively as; Baek and Kim [7].

The drag coefficient $\mathrm{Cd}$ changes in accordance with the size, depth and number of dimples in a golf ball. The coefficient for the number of dimples is determined using the interpolation of the experimentally given values to produce a hyperbolic function which is a function of critical Reynolds number and varied Reynolds's number as below.

$$
C_{d(\text { number })}=\tanh \left[\frac{\mathrm{Re}^{*}}{\operatorname{Re} \times \text { no.of dim ples }}-0.05\right]
$$

The coefficient for the dimple size is consituted from a linear interpolation from experimental data subject to the ratio of dimple size to the ball diameter [9].

$$
C_{d(\text { size })}=0.25-2.046 \frac{c}{d}
$$

Where $\mathrm{Re} *=1 \times 107$ and $\mathrm{Re}=3 \times 105$ are the critical and varied Reynolds number respectively. Usually the drag coefficient has deep connections with this dimensionless number [9].

The coefficient for the dimple depth is also obtained from a linear interpolation of the same experimental data.

$$
\begin{aligned}
& C_{d(\text { depth })}=1.2-\cos \left[100\left(0.005-\frac{k}{d}\right)\right] \\
& C_{l(\text { depth })}=1.2-\cos \left[100\left(0.005-\frac{k}{d}\right)\right]
\end{aligned}
$$

$\mathrm{Cl}($ number $)=$ norm.pdf $(\operatorname{Re}, 200 \times$ no. of dimples,9000 $)$

The $\mathrm{Cl}$ for dimple number was obtained by Bearman and Harvey [9] as a normal probabiliy distribution function with the mean being the value $200 \times$ no. of dimples and the standard deviation as 9000 .

Here $\ldots$ indicates the ratio of dimple diameter to the golf ball diameter and ....... is the ratio of dimple depth to the golf ball diameter.

Having obtained the above eqns. (23 and 25) that model the motion of a golf ball in 3 dimensions; we then present, in the next section, the methods of solving these equations so as to obtain the flight trajectory and distances travelled along the golf fairway.

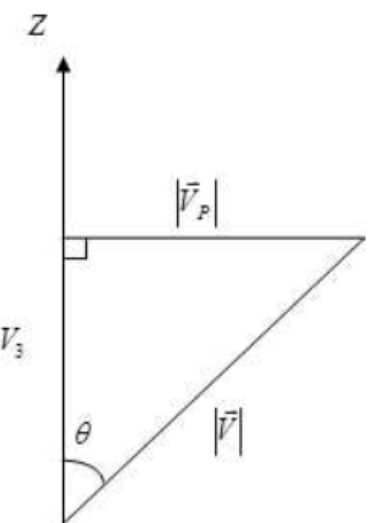

Figure 7: Representation of the velocity vector components, its magnitude and projection in 2 dimensions. 


\section{Method of Solution}

\section{The model equations in non-dimensional form}

To obtain the non-dimensional version of the eqns. (23 and 25) we note that the dimensions contained in our variables and parameters are lengths and time. For our case we choose the following relations for our lengths and time,

$$
X=\bar{L}_{1} x, Y=\bar{L}_{2} y, Z=\bar{L}_{3} z, t=\tau T
$$

Where $\bar{L}_{1}, \bar{L}_{2}$ are the characteristic lengths in the dimensionless $\mathrm{x}$ and $\mathrm{y}$ directions to the target place, which can either be to the hole or a layup distance. The other characteristic length ${ }^{L_{3}}$ in the dimensionless $\mathrm{z}$ direction is expressed in terms of the characteristic time and magnitude of gravity fopce $\bar{L}_{s} L_{3}=g \tau^{2}$. The characteristic speed can also be determined as $\tau$ since for a ball in flight, the velocity in the $y$ direction must be significantly higher than that in the $\mathrm{x}$ and $\mathrm{z}$ directions, that is the ball travels furthest in the $\mathrm{y}$ direction to the expectation of the player. In view of this, we assume that the ball is teed primarily in the y direction, thus $L_{1}<<L_{2}$. Now using the relation in non-dimensional parameter above the equations of motion in component form becomes

$$
\begin{aligned}
& \ddot{x} \frac{\bar{L}_{1}}{\tau^{2}}=-K_{d}|\vec{V}| \dot{x} \frac{\overline{L_{1}}}{\tau}+k_{L} \frac{|\vec{V}|}{|\vec{V}|} \dot{x} \dot{z} \frac{\bar{L}_{1}}{\tau} \frac{\bar{L}_{3}}{\tau} \\
& \ddot{y} \frac{\bar{L}_{2}}{\tau^{2}}=-K_{d}|\vec{V}| \dot{Y} \frac{\bar{L}_{2}}{\tau}+k_{L} \frac{|\vec{V}|}{\left|\overrightarrow{V_{P}}\right|} \dot{y} \dot{z} \frac{\bar{L}_{2}}{\tau} \frac{\bar{L}_{3}}{\tau} \\
& \ddot{z} \frac{\bar{L}_{3}}{\tau^{2}}=-g-K_{d}|\vec{V}| \dot{z} \frac{\bar{L}_{3}}{\tau}+k_{L}|\vec{V}||\vec{V}|
\end{aligned}
$$

Next we express the magnitudes of velocity and its projection in terms of the characteristic length and time as follows:

$$
\begin{aligned}
& |\vec{V}|=\sqrt{\left(\dot{X}^{2}+\dot{Y}^{2}+\dot{Z}^{2}\right)}=\sqrt{\left(\dot{x}^{2} \frac{\bar{L}_{1}^{2}}{\tau^{2}}+\dot{y}^{2} \frac{\bar{L}_{2}^{2}}{\tau^{2}}+\dot{z}^{2} \frac{\bar{L}_{3}^{2}}{\tau^{2}}\right)} \\
& |\vec{V}|=\sqrt{\left(\dot{X}^{2}+\dot{Y}^{2}\right)}=\sqrt{\left(\dot{x}^{2} \frac{\bar{L}_{1}^{2}}{\tau^{2}}+\dot{y}^{2} \frac{\bar{L}_{2}^{2}}{\tau^{2}}\right)}
\end{aligned}
$$

Factoring out $\frac{\dot{y} L_{2}}{\tau}$

$$
\begin{aligned}
& |\vec{V}|=\frac{\dot{y} \bar{L}_{2}}{\tau} \sqrt{\dot{x}^{2} \frac{\bar{L}_{1}^{2}}{\dot{y}^{2} \bar{L}_{2}^{2}}+1+\dot{z}^{2} \frac{\bar{L}_{3}^{2}}{\dot{y}^{2} \bar{L}_{2}^{2}}}=\frac{\dot{y} \bar{L}_{2}}{\tau} r_{1} \\
& \left|\vec{V}_{p}\right|=\frac{\dot{y} L_{2}}{\tau} \sqrt{\dot{x}^{2} \frac{\bar{L}_{1}^{2}}{\dot{y}^{2} \bar{L}_{2}^{2}}+1}=\frac{\dot{y} \bar{L}_{2}}{\tau} r_{2}
\end{aligned}
$$

$$
\text { Where } r_{1}=\sqrt{\left(\dot{x}^{2} \frac{\overline{L_{1}^{2}}}{\dot{y}^{2} \bar{L}_{2}^{2}}+1+\dot{z}^{2} \frac{\overline{L_{3}^{2}}}{\dot{y}^{2} \bar{L}_{2}^{2}}\right)} \text { and } r_{2}=\sqrt{\dot{x}^{2} \frac{\overline{L_{1}^{2}}}{\dot{y}^{2} \overline{L_{2}^{2}}}+1}
$$

Now using the eqns. (38) and (39) in the eqns. (34 and 36) we obtain the following set of non-dimensional equations

$$
\begin{aligned}
& \ddot{x}=-K_{d} \bar{L}_{2} \dot{x} \dot{y} r_{1}+K_{l} \bar{L}_{3} \frac{r_{1}}{r_{2}} \dot{x} \dot{z} \\
& \ddot{y}=-K_{d} \bar{L}_{2} \dot{y}^{2} r_{1}+K_{l} y \bar{L}_{3} \frac{r_{1}}{r_{2}} \dot{y} \dot{z}
\end{aligned}
$$

$$
\ddot{z}=-1-K_{d} \bar{L}_{2} r_{1} \dot{y} \dot{z}-K_{L} \frac{\bar{L}_{2}^{2}}{\bar{L}_{3}} r \dot{y}^{2}
$$

Next we write the system of eqns. (40)-(42) in a vector form, suitable for the subsequent sections. Let us define $\vec{R}$ and $\vec{F}(t, \vec{R})$ as the column vectors:

$$
\begin{aligned}
& \vec{R}(t)=\left[\begin{array}{c}
\dot{x}(t) \\
\dot{y}(t) \\
\dot{z}(t)
\end{array}\right], \vec{F}(t, \vec{R})=\left[\begin{array}{l}
f_{1}(\dot{x}(t), \dot{y}(t), \dot{z}(t)) \\
f_{2}(\dot{x}(t), \dot{y}(t), \dot{z}(t)) \\
f_{3}(\dot{x}(t), \dot{y}(t), \dot{z}(t))
\end{array}\right] \\
& f_{1}(\dot{x}(t), \dot{y}(t), \dot{z}(t))=-\dot{y} r_{1}\left(K_{d} \bar{L}_{2} \dot{x}+K_{l} \bar{L}_{3} \frac{\dot{x} \dot{z}}{r_{2} \dot{y}}\right) \\
& f_{2}(\dot{x}(t), \dot{y}(t), \dot{z}(t))=-\dot{y} r_{1}\left(K_{d} \bar{L}_{2} \dot{y}+K_{l} \bar{L}_{3} \frac{\dot{z}}{r_{2}}\right) \\
& f_{3}(\dot{x}(t), \dot{y}(t), \dot{z}(t))=-1-\dot{y} r_{1}\left(K_{d} \overline{L_{2}} \dot{z}+K_{l} \frac{L_{2}}{L_{3}} r_{2} \dot{y}\right)
\end{aligned}
$$

Thus using the above column vectors together with relations (43) the system of equations becomes:

$$
\frac{d(\vec{R}(t))}{d t}=\vec{F}(t, \vec{R})
$$

Subject to the initial conditions $\vec{R}(t)=\left(\dot{x}\left(t_{0}\right), \dot{y}\left(t_{0}\right), \dot{z}\left(t_{0}\right)\right)$ where the initial time is denoted by $\mathrm{t}_{0}$.

The Dormand-Prince Runge-Kutta method is used to numerically solve the modelled equations as outlined. The coefficients in this method are chosen in a manner that minimizes the error of the fifth order solution, which is an advancement of the known Feldberg RungeKutta method that was constructed to minimize the error of the fourth order solution. This makes the Dormand-Prince method more suitable when the higher order solution is used to continue the integration, a practice known as local integration [10].

\section{Results and Discussions}

\section{Effects of different dimple number on the flight trajectory}

Dimples affect the lift of a golf ball and therefore its trajectory. While the ball spin contributes one part of a golf ball's lift, the other part is provided by the dimples, and both are vital for optimization of the lift. In the previous chapters, it was stated that dimples improve the aerodynamic characteristics and flight distance of the ball. Typical golf balls have between 300-500 dimples. The reason for the large number of dimples is to ensure that the boundary layer does not separate until the back part of the ball. Additionally, the high number of dimples is desirable due to the increased turbulence created which will consequently reduce drag on the ball and improve the distance the ball can travel (Figure 8).

We simulate the distances achieved by different dimple characteristics using the initial conditions.

$$
\begin{aligned}
& \{\mathrm{x}(0), \mathrm{y}(0), \mathrm{z}(0)\}=\{0,0,0\} \\
& \left\{V_{x}(0), V_{y}(0), V_{z}(0)\right\}=\left\{0,1, \frac{\tau \omega}{L_{3}}\right\}, \tau=\frac{L_{2}}{v_{0}}, \omega=120
\end{aligned}
$$

These same initial conditions are used in the subsequent sections. In Figures 9 and 10 it can be seen that the ball with the least number of dimples (250) has a flight path that is much like a flop i.e., a short trajectory used to get the ball over an obstacle and stops very quickly, 


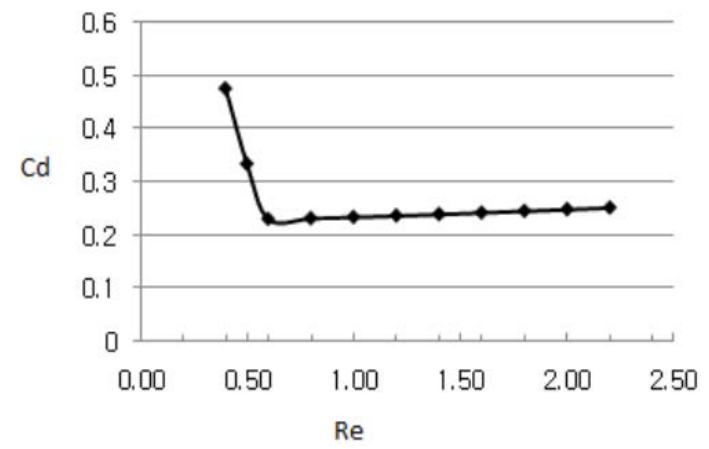

Figure 8: Drag coefficient for different Reynolds numbers (Seongman et al. (2013)).

it is characterized by a higher loft. The ball with 330 dimples has a relatively shorter distance than the one with 450 dimples, which indeed shows that the developed model is able to reproduce what happens in reality.

As we compare the ball trajectories with different number of dimples here, note that other factors like the depth and size of the dimple are held constant. We presume this is the reason for the marginal differences in horizontal distances and loft where max $\sqrt{X^{2}+Y^{2}}$ is the projection

\section{Effect of dimple size $\left(\frac{c}{d}\right)$ on the flight trajectory}

The effect of dimple size is measured by the ratio of dimple diameter to the ball diameter $\left(\frac{c}{d}\right)$. The results are presented in Figures 11 and 12 for $\frac{c}{d}=0.06, \frac{c}{d}=0.06, \frac{c}{d}=0.1$. The ratio $\frac{c}{d}=0.03$ reflects a ball with deep dimples and indicates that it is capable of achieving longer distances as compared to the other ratios $\frac{c}{d}=0.03$, and $\frac{c}{d}=0.03$. Smaller dimples $\frac{c}{d}=0.03$ generate more back-spin on a golf ball than bigger dimples $\frac{c}{d}=0.06, \frac{c}{d}=0.1$ which increases lift and cause the ball to rise higher but fails to generate sufficient turbulence thus the ball drops faster as shown in Figure 11. The ratio $\frac{c}{d}=0.1$, registers the longest distance (Figures 11 and 12). This is because if the size of the dimples is large its drag effect diminishes thus keeping the ball in the air for longer. If the number of dimples is increased then their size must get smaller and so the ball becomes almost smooth and will not perform very well in acquiring the required loft and carry. Therefore a proper trade-off between dimple number and size must be arrived at.

The results obtained here for bigger dimple size are quite comparable with those achieved by Naruo and Mizota [5] for $\frac{c}{d}=0.08$.

Effect of dimple depth $\left(\frac{k}{d}\right)$ on the flight trajectory

We already know that dimples on the ball are responsible for the reduction in drag. The lift and drag forces on a golf ball are very sensitive to dimple depth since a depth of 0.001 inches can produce a radical change to the balls trajectory and consequently the overall distance it can fly. Ideally, a standard dimple measures approximately $7 / 1000^{\text {th }}$ of an inch deep. Adding or subtracting 1/1000 the results to a change in distance with plus or minus 5 yards. Sajima [11] considered the influence of the dimple depth using CFD. In his study the influence of dimples was systemized and investigated experimentally using wind tunnel and the ball rotation device. Consequently it was found that the influence of the dimple depth was significant and the shallower the dimple the more the lift coefficient and it gained a higher trajectory. However, shallowness exceeding a certain threshold, the lift coefficient declined greatly in the low speed hence achieving a short distance.

In this study we consider the measurement of dimple depth as the ratio of dimple depth to the ball diameter as a dimensionless coefficient and vary it as $\frac{k}{d}=0.006, \frac{k}{d}=0.006, \frac{k}{d}=0.012$ while holding other parameters constant. Our results in Figure 13 show that the highest trajectory is achieved by the ratio $\frac{k}{d}=0.003$ that represents a deeper dimple. And the distance it achieves is the shortest. However, to any

$k / d=0.006, R e=3^{*} 10^{5}, c / d=0.06$

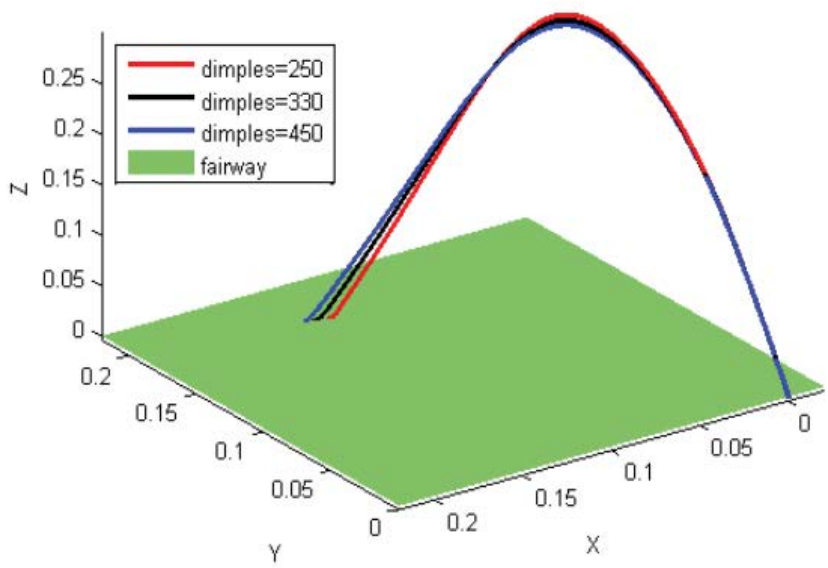

Figure 9: Three dimension depiction of flight trajectories for varied dimple number.

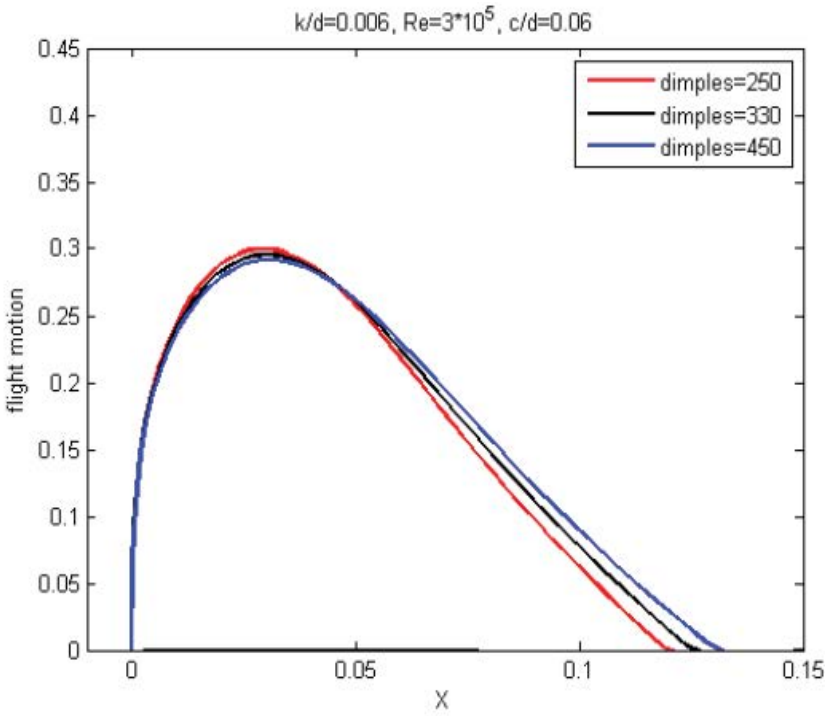

Figure 10: Flight trajectory for varied dimple numbers as observed along the $x$-axis where the $z$ axis has been re-labelled flight motion to indicate the loft of the flight trajectory on the $X-Y$ plane. 
$k / d=0.006, R \theta=3 * 10^{5}$, dimples $=330$

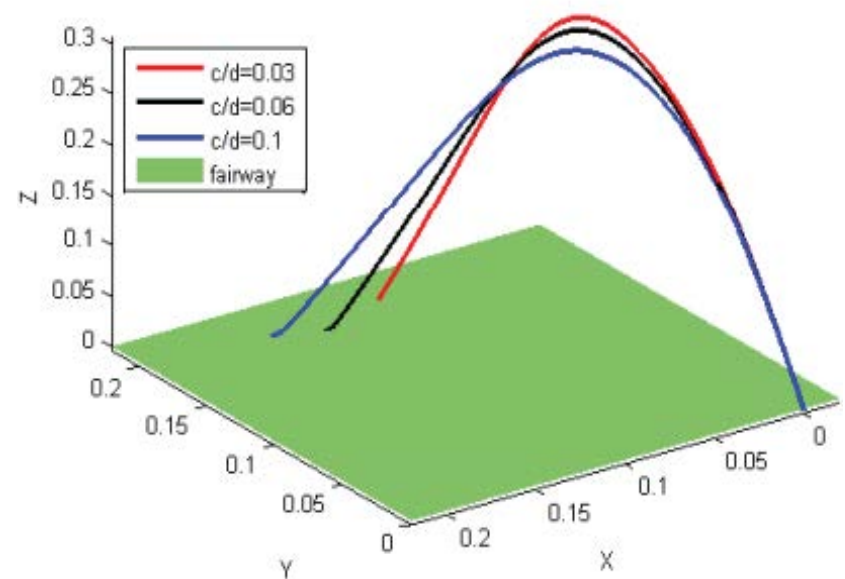

Figure 11: Three dimensional depictions of flight trajectories for varied dimple size.

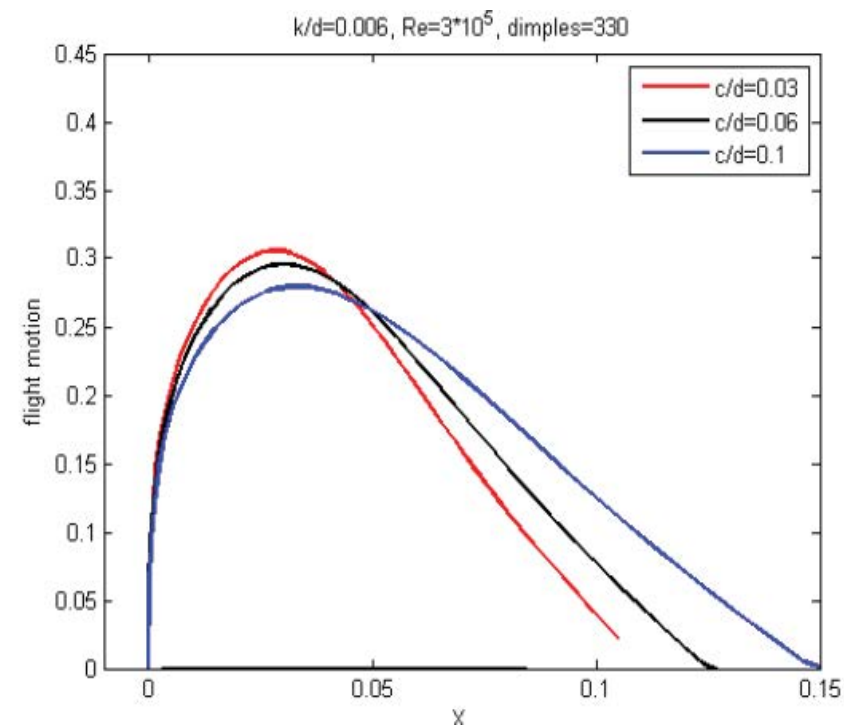

Figure 12: Flight trajectories for varied dimple size as viewed along the $x$-axis where the $z$ axis has been re-labelled flight motion to indicate the loft.

golfer, distance is a very desirable aspect and anything working for greater distance is most acknowledged. As the depth increases the drag coefficient increases resulting to poor air resistance and therefore premature landing of the ball. On the other hand the ratios $\frac{k}{d}=0.003$ ,$\frac{k}{d}=0.006$ achieve high trajectory and longer distance, with the moderately shallower dimples having the longest distance (Figure 14). This agrees with the study in Ting [12], Baek and Kim [7] and Mehta and Pallis [13].

\section{Conclusions and Recommendations}

In conclusion, the mathematical simulation of the dimple characteristics reveal that the aerodynamic behaviour and trajectory of the golf ball in flight is primarily dependent on the physical features of dimples. $c / d=0.06, R e=3^{*} 10^{5}$, dimples $=330$

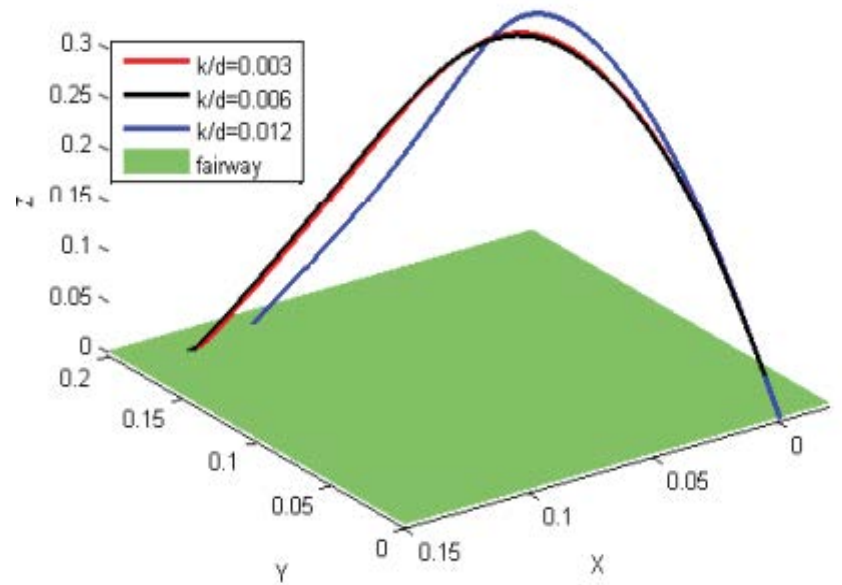

Figure 13: A simulation of the effect of dimple depth on the flight trajectory.

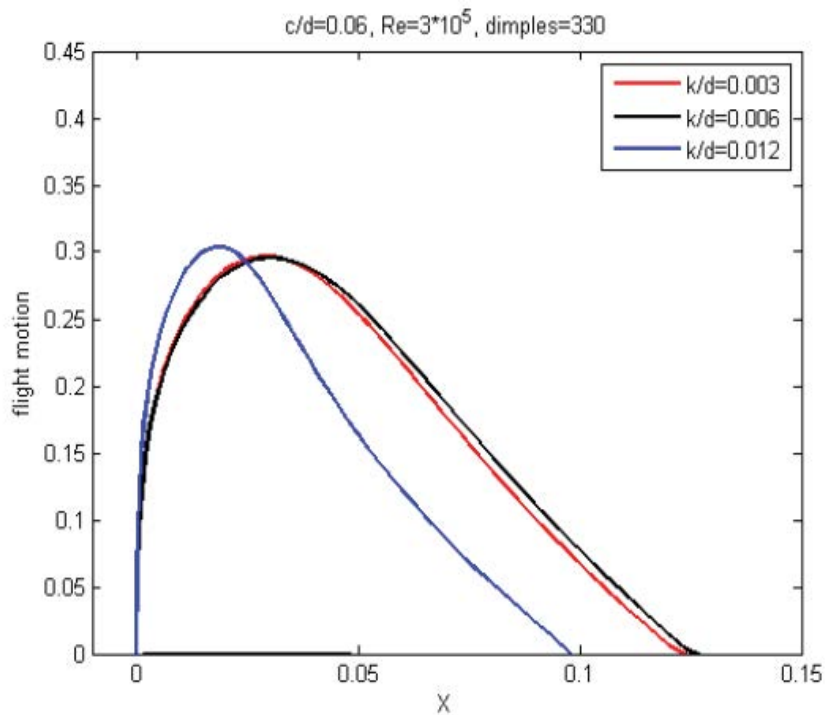

Figure 14: Depiction of the simulation results in Fig. 13 along the $x$-axis where the $z$ axis has been re-labelled flight motion to indicate the loft.

By changing the sign of from positive to negative in the developed model enabled us to reproduce the trajectory of a ball hit with back-and top-spin respectively.

This study considered the motion of the golf ball from the instance of take off to the point when it lands. Future studies can include the roll that happens once the ball lands and what factors are responsible for a big or small roll. Moreover, future study can include putting as this important stage of scoring to a golfer.

\section{Acknowledgement}

I wish to express my appreciation and gratitude to my supervisors Dr. Kimath and Prof. Malonza for their help and encouragement in the entire during of writing this thesis. I also appreciate my classmate now Dr. Sr. Njeri for the encouragement to soldier on even when the going got tough. 
Citation: Wainaina M, Kimathi M, Malonza D (2018) Simulation of Dimple Characteristics on the Trajectories of a Dimpled Sphere (Golf Ball) in Motion. J Appl Computat Math 7: 386. doi: 10.4172/2168-9679.1000386

Page 9 of 9

\section{References}

1. Aoki K, Muto K, Okanaga H, Nakayama Y (2009) Aerodynamic characteristic and flow pattern on dimples structure of a sphere. Flucome 10th International Conference on Fluid Control, Measurement and Visualization.

2. Aoki K, Muto K, Okanaga H (2010) Aerodynamic characteristics and flow pattern of a golf ball with rotation. Procedia Engineering 2: 2431-2436.

3. Aoki K, Nonaka M, Goto T. Aerodynamics and flying characteristic for the surface structure of the golf ball. Proceedings of the School of Engineering Tokyo University 44.

4. Aoki K, Ohike A, Yamaguchi K, Nakayama Y (2003) Flying characteristics and flow pattern of a sphere with dimples. Journal of visualization 6: 67-76.

5. Naruo T, Mizota T (2014) The influence of golf ball dimples on aerodynamic characteristics. Procedia Engineering 72: 780-785

6. Myers TG, Mitchell SL (2013) A mathematical analysis of the motion of an inflight soccer ball. Sports Engineering 16: 29-41.
7. Baek S, Kim M (2013) Flight trajectory of a golf ball for a realistic game. International Journal of Innovation, Management and Technology 4: 346.

8. Alam F, Steiner T, Chowdhury H, Moria H, Khan I, et al. (2011). A study of golf ball aerodynamic drag. Procedia Engineering 13: 226-231.

9. Bearman PW, Harvey JK (1976) Golf ball aerodynamics. The Aeronautical Quarterly 27: 112-122.

10. Gander MJ, Hairer E (2008) Nonlinear convergence analysis for the parareal algorithm. In: Domain Decomposition Methods in Science and Engineering XVII, pp: 45-56.

11. Sajima T (2006) Golf Ball. US Patent.

12. Ting LL (2003) Effects of dimple size and depth on golf ball aerodynamic performance. In: ASME/JSME 2003 4th Joint Fluids Summer Engineering Conference, pp: 811-817.

13. Mehta RD, Pallis JM (2001) Sports ball aerodynamics: effects of velocity spin and surface roughness. Minerals, Metals and Materials Society/AIME Materials and Science in Sports, pp: 185-197. 